\title{
Experimental comparison of ring and diamond shaped planar Hall effect bridge magnetic field sensors
}

\author{
Henriksen, Anders Dahl; Rizzi, Giovanni; Hansen, Mikkel Fougt
}

Published in:

Journal of Applied Physics

Link to article, DOI:

$10.1063 / 1.4930068$

Publication date:

2015

Document Version

Publisher's PDF, also known as Version of record

Link back to DTU Orbit

Citation (APA):

Henriksen, A. D., Rizzi, G., \& Hansen, M. F. (2015). Experimental comparison of ring and diamond shaped planar Hall effect bridge magnetic field sensors. Journal of Applied Physics, 118(10), [103901].

https://doi.org/10.1063/1.4930068

\section{General rights}

Copyright and moral rights for the publications made accessible in the public portal are retained by the authors and/or other copyright owners and it is a condition of accessing publications that users recognise and abide by the legal requirements associated with these rights.

- Users may download and print one copy of any publication from the public portal for the purpose of private study or research.

- You may not further distribute the material or use it for any profit-making activity or commercial gain

- You may freely distribute the URL identifying the publication in the public portal 


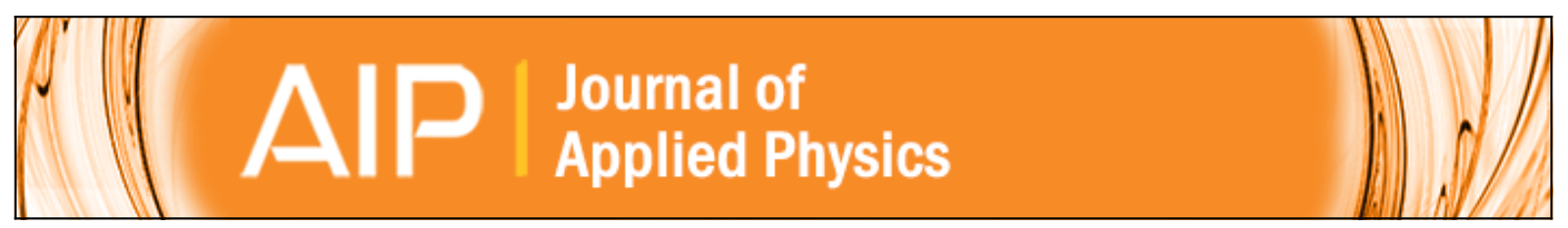

\section{Experimental comparison of ring and diamond shaped planar Hall effect bridge magnetic field sensors}

Anders Dahl Henriksen, Giovanni Rizzi, and Mikkel Fougt Hansen

Citation: Journal of Applied Physics 118, 103901 (2015); doi: 10.1063/1.4930068

View online: http://dx.doi.org/10.1063/1.4930068

View Table of Contents: http://scitation.aip.org/content/aip/journal/jap/118/10?ver=pdfcov

Published by the AIP Publishing

\section{Articles you may be interested in}

Comment on "Planar Hall resistance ring sensor based on NiFe/Cu/IrMn trilayer structure" [J. Appl. Phys. 113, 063903 (2013)]

J. Appl. Phys. 114, 106101 (2013); 10.1063/1.4820925

Erratum: "Planar Hall resistance ring sensor based on NiFe/Cu/IrMn trilayer structure" [J. Appl. Phys. 113, 063903 (2013)]

J. Appl. Phys. 114, 089902 (2013); 10.1063/1.4819893

Planar Hall resistance ring sensor based on $\mathrm{NiFe} / \mathrm{Cu} / \mathrm{lrMn}$ trilayer structure

J. Appl. Phys. 113, 063903 (2013); 10.1063/1.4790139

High field-sensitivity planar Hall sensor based on NiFe/Cu/lrMn trilayer structure

J. Appl. Phys. 107, 09E715 (2010); 10.1063/1.3337739

Planar Hall bead array counter microchip with NiFe/lrMn bilayers

J. Appl. Phys. 104, 074701 (2008); 10.1063/1.2988297

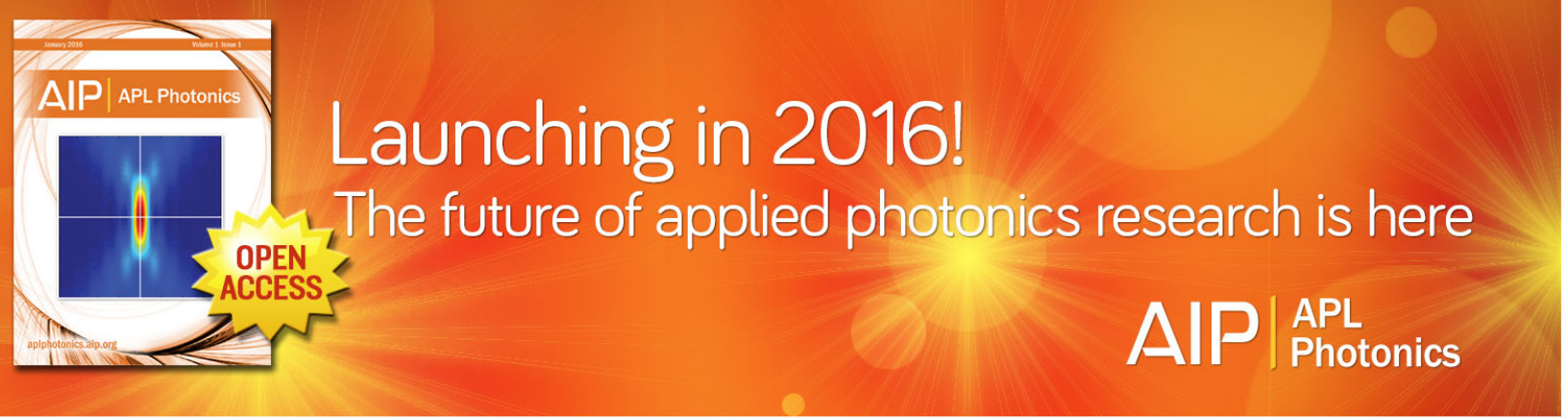




\title{
Experimental comparison of ring and diamond shaped planar Hall effect bridge magnetic field sensors
}

\author{
Anders Dahl Henriksen, Giovanni Rizzi, and Mikkel Fougt Hansen a) \\ Department of Micro- and Nanotechnology, Technical University of Denmark, DTU Nanotech, \\ Building 345 East, DK-2800 Kongens Lyngby, Denmark
}

(Received 10 July 2015; accepted 24 August 2015; published online 8 September 2015)

\begin{abstract}
Planar Hall effect magnetic field sensors with ring and diamond shaped geometries are experimentally compared with respect to their magnetic field sensitivity and total signal variation. Theoretically, diamond shaped sensors are predicted to be $41 \%$ more sensitive than corresponding ring shaped sensors for negligible shape anisotropy. To experimentally validate this, we have fabricated both sensor geometries in the exchange-biased stack $\mathrm{Ni}_{80} \mathrm{Fe}_{20}\left(t_{\mathrm{FM}}\right) / \mathrm{Cu}\left(t_{\mathrm{Cu}}\right) /$ $\mathrm{Mn}_{80} \mathrm{Ir}_{20}(10 \mathrm{~nm})$ with $t_{\mathrm{FM}}=10,20$, and $30 \mathrm{~nm}$ and $t_{\mathrm{Cu}}=0,0.3$, and $0.6 \mathrm{~nm}$. Sensors from each stack were characterized by external magnetic field sweeps, which were analyzed in terms of a single domain model. The total signal variation of the diamond sensors was generally found to be about $40 \%$ higher than that for the ring sensors in agreement with theoretical predictions. However, for the low-field sensitivity, the corresponding improvement varied from $0 \%$ to $35 \%$ where the largest improvement was observed for sensor stacks with comparatively strong exchange bias. This is explained by the ring sensors being less affected by shape anisotropy than the diamond sensors. To study the effect of shape anisotropy, we also characterized sensors that were surrounded by the magnetic stack with a small gap of $3 \mu \mathrm{m}$. These sensors were found to be less affected by shape anisotropy and thus showed higher low-field sensitivities. (C) 2015 AIP Publishing LLC.
\end{abstract}

[http://dx.doi.org/10.1063/1.4930068]

\section{INTRODUCTION}

Magnetic field sensors based on giant magneto-resistance, ${ }^{1,2}$ magnetic tunneling resistance, ${ }^{3,4}$ and anisotropic magnetoresistance ${ }^{5-8}$ effects have been presented in the literature with special focus on their application for magnetic biosensing. ${ }^{9}$ For such applications, magnetic beads are used as readout labels and are linked to the surface of the sensor chip in the presence of the target analyte. Such sensors also have important applications, e.g., in compasses and for the non-contact monitoring of currents. ${ }^{10}$

For magnetoresistive sensors, where the readout relies on the planar Hall effect in an exchange-biased magnetic thin film, the sensor design paradigm shifted in 2010 from a single resistor element found in cross-shaped planar Hall effect sensors ${ }^{11-13}$ to Wheatstone bridge designs, ${ }^{6,14,15}$ which provided higher resistance and sensitivity while still having nominally zero offset. These designs, termed planar Hall effect bridge (PHEB) sensors, also greatly expanded the sensor design space. For example, we have described a building block approach to the sensor design and its optimization for magnetic bead detection. ${ }^{16}$ Also, the use of PHEB sensors for both volume- and surface-based biodetection schemes using only the field from the sensor bias current as magnetic field excitation has been demonstrated. ${ }^{17-19}$

While these PHEB sensors showed improved signal-tonoise ratio, both ring shaped sensors with curved resistors ("ring sensors") 7,15 and diamond shaped sensors with straight resistors ("diamond sensors") ${ }^{14,20}$ have been argued to be the

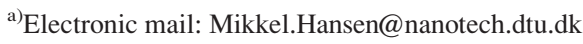

superior design. The pros and cons of ring and diamond sensors were recently discussed theoretically for the detection of external magnetic fields and a theoretical analysis predicted the diamond sensors to be $41 \%$ more sensitive than the corresponding ring sensors for the same bias current. ${ }^{20}$

Here, we perform a systematic experimental comparison of the signals from ring and diamond sensors when these are used to measure externally applied magnetic fields. Sweeps of the sensor response vs. applied magnetic field are analyzed using a single domain magnetic energy model, which is used to extract and compare important sensor parameters for a range of magnetic stack compositions. Moreover, we investigate the impact of introducing a magnetic stack surrounding the sensors to reduce the shape anisotropy of the sensors.

\section{THEORY}

\section{A. Sensor geometries}

The sensors in this study are all anisotropic magnetoresistance/PHEB sensors with four resistors in a Wheatstone bridge configuration. ${ }^{14}$ Figure 1 illustrates the four sensor geometries included in the study. Two of the sensors have a diamond geometry with straight resistive elements of dimensions $l \times w=250 \mu \mathrm{m} \times 25 \mu \mathrm{m}$ and two have a ring geometry with curved resistive elements of radius $r=l / \sqrt{2}$ and width $w$. The relation between $l$ and $r$ was chosen to have the electrical contacts at the same locations for the two designs, i.e., to have the same nominal footprint of the two sensors. For both the ring and diamond sensors, two versions were studied: (1) a simple bare sensor geometry not surrounded 

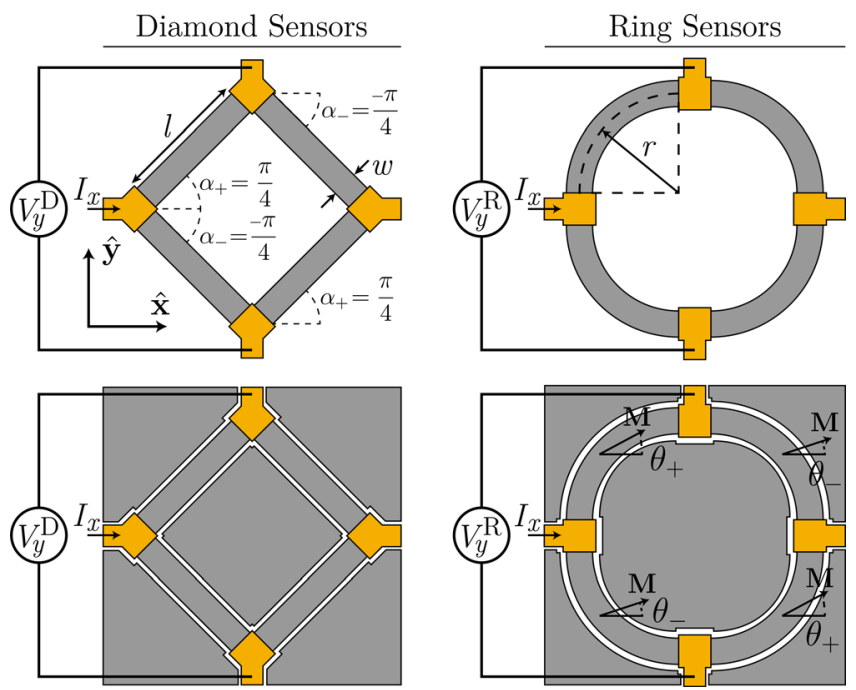

FIG. 1. Illustration of the diamond (left) and ring (right) sensor geometries with definitions of parameters and coordinate system (not to scale). The dark grey and orange colors indicate the magnetic stack and the contact layer, respectively. The magnetization angle of the sensor arms forming positive and negative angles to the $x$-axis are denoted $\theta_{ \pm}$, respectively. The top bare sensors are not surrounded by magnetic stack. The bottom surrounded sensors are surrounded by magnetic stack with a gap of $3 \mu \mathrm{m}$.

by any magnetic material (top row) and (2) a surrounded sensor geometry where the sensor is surrounded by the magnetic stack with a gap of $3 \mu \mathrm{m}$ (bottom row).

\section{B. Sensor signal vs. magnetization angles}

Letting $\theta_{+}$and $\theta_{-}$denote the respective equivalent single domain angles of the magnetization to the $x$-axis for the bridge branches with positive and negative slopes in Fig. 1, the bridge output signals for the ring (R) and diamond (D) sensors can be calculated as ${ }^{20}$

$$
\begin{aligned}
& V_{y}^{\mathrm{R}}=\frac{r}{4 w t} I_{x} \Delta \rho\left[\sin \left(2 \theta_{+}\right)+\sin \left(2 \theta_{-}\right)\right], \\
& V_{y}^{\mathrm{D}}=\frac{l}{4 w t} I_{x} \Delta \rho\left[\sin \left(2 \theta_{+}\right)+\sin \left(2 \theta_{-}\right)\right] .
\end{aligned}
$$

Here, $t$ is the thickness of the magnetic stack, $I_{x}$ is the current applied through the sensor, and $\Delta \rho=\rho_{\|}-\rho_{\perp}$ is the difference in stack resistivity when the current and magnetization vectors are parallel and perpendicular, respectively. In this work, we have chosen the sign convention of Eqs. (1) and (2) such that positive signals are obtained for small positive values of $\theta_{ \pm}$, i.e., the values are given as voltage drops over the sensor bridge in the $y$-direction.

The maximum and minimum signals are obtained when $\theta_{ \pm}=45^{\circ}$ and $\theta_{ \pm}=-45^{\circ}$, respectively, and they span a range $V_{\mathrm{pp}}$, which is characteristic for the sensor geometry. When the sensor contacts are placed at identical positions, $l=\sqrt{2} r$, and the signal spans for the diamond and ring sensors are related as $V_{\mathrm{pp}}^{\mathrm{D}}=\sqrt{2} V_{\mathrm{pp}}^{\mathrm{R}}$, i.e., the output from the diamond sensor is about $41 \%$ higher than that from the equivalent ring sensor.

Moreover, the resistance of the sensor bridge, $R_{\mathrm{b}}$, measured along the $x$-axis is independent of the magnetization angle $\theta_{ \pm}$and the bridge resistances for the two designs are related as $R_{\mathrm{b}}^{\mathrm{R}}=R_{\mathrm{b}}^{\mathrm{D}} \pi /(2 \sqrt{2}) \approx 1.11 R_{\mathrm{b}}^{\mathrm{D}}$, i.e., the bridge resistance for the ring sensor is $11 \%$ higher than that for the diamond sensor.

\section{Magnetic energy}

All sensors were based on permalloy, which is a ferromagnetic (FM) material displaying anisotropic magnetoresistance. The magnetization of the FM layer was exchange-pinned along the $x$-direction using an antiferromagnetic (AFM) layer. Some sensors also included a thin copper layer between the FM and AFM layers to weaken the exchange bias coupling. ${ }^{7}$ Upon application of a magnetic field $B_{y}$ along the $y$-direction, the equivalent single domain magnetization angle $\theta_{ \pm}$for a sensor branch can be obtained by minimizing the energy density, $u$. The energy density normalized with the saturation magnetization $M_{s}$ of the ferromagnetic layer is

$$
\begin{aligned}
\tilde{u}_{ \pm}= & \frac{u}{M_{s}}=-B_{y} \sin \theta_{ \pm}-B_{\mathrm{ex}} \cos \theta_{ \pm} \\
& -\frac{1}{2} B_{\mathrm{K}} \cos ^{2} \theta_{ \pm}-\frac{1}{2} B_{\mathrm{sh}} \cos ^{2}\left(\theta_{ \pm}-\alpha_{ \pm}\right) .
\end{aligned}
$$

Here, $B_{\mathrm{ex}}$ is the exchange bias field, $B_{\mathrm{K}}$ is the anisotropy field, $B_{\text {sh }}$ is the shape anisotropy field, and $\alpha_{ \pm}$are the respective angles of the current to the $x$-axis for the branches with positive and negative slopes. For the diamond sensor, we have exactly that $\alpha_{ \pm}= \pm 45^{\circ}$, whereas for the ring sensor, we will assume $\alpha_{ \pm}= \pm 45^{\circ}$ in an equivalent single domain description of the magnetization of the sensor branches. This assumption enables direct comparison of parameters obtained for the various sensor designs by minimization of Eq. (3).

For negligible shape anisotropy, $\theta_{+}=\theta_{-}=\theta$ and low magnetic fields the solution that minimizes Eq. (3) is

$$
\theta \approx \frac{B_{y}}{B_{\mathrm{ex}}+B_{\mathrm{K}}} .
$$

A high value of $B_{\mathrm{ex}}$ weakens the relative influence of the applied field leading to a smaller sensitivity. The conditions for maximum and minimum sensor output, $\theta= \pm 45^{\circ}$, are obtained for external fields of $B_{\text {peak }}= \pm\left(B_{\mathrm{ex}}+B_{\mathrm{K}} / \sqrt{2}\right)$. Moreover, the low-field sensitivities for the two designs are defined as

$$
S_{0}^{\mathrm{R}, \mathrm{D}}=\left.\frac{\partial V_{y}^{\mathrm{R}, \mathrm{D}}}{I_{x} \partial B_{y}}\right|_{B_{y}=0} .
$$

From Eqs. (1)-(5), it is observed that the diamond sensors are expected to be $41 \%$ more sensitive than the corresponding ring sensors for negligible shape anisotropy.

\section{Impact of shape anisotropy}

Shape anisotropy originates from the increase in magnetic energy when an object is magnetized along its shorter direction. Mathematically, this is described by the demagnetization field $\mathbf{H}_{\mathrm{d}}$ and the demagnetization tensor $\underline{\underline{N}}$ as $\mathbf{H}_{\mathrm{d}}=-\underline{N} \mathbf{M}$, where $\mathbf{M}$ is the magnetization vector. This 
description is exact for ellipsoids and approximately valid for prisms such as the magnetic stack in a sensor arm. For thin films, the shape anisotropy confines the magnetization to the $x y$-plane. For this case, the shape anisotropy field in Eq. (3) is given by $B_{\text {sh }}=\mu_{0} M_{s}\left(N_{\perp}-N_{\|}\right)$, where $N_{\perp}$ and $N_{\|}$ are the in-plane demagnetization factors perpendicular and parallel to a sensor arm, respectively.

The shape anisotropy energy is lowest when the magnetization is along the long axis of a sensor arm and hence affects the resistors with $\alpha_{+}=+45^{\circ}$ differently from those with $\alpha_{-}=-45^{\circ}$. This makes the sensor output more complicated if the sensor has non-negligible shape anisotropy. Figure 2 shows plots of the normalized sensor output for different values of the shape anisotropy field calculated by minimizing Eq. (3) to find $\theta_{+}$and $\theta_{-}$and inserting into Eq. (2). For increasing values of $B_{\mathrm{sh}}<B_{\mathrm{ex}}$, the low-field sensitivity decreases but both the positions of the peaks and the peak-to-peak voltage $V_{\mathrm{pp}}$ remain constant. Moreover, the response curve is observed to assume a characteristic S-like shape with progressively sharper features near $\pm B_{\text {peak }}$. Values of $B_{\mathrm{sh}}>B_{\text {ex }}$ result in a decrease of $V_{\mathrm{pp}}$ and the sensor response may also become hysteretic.

\section{EXPERIMENTAL}

\section{A. Sensor fabrication}

Sensors with the stack $\mathrm{Ta}(13 \mathrm{~nm}) / \mathrm{Ni}_{80} \mathrm{Fe}_{20}\left(t_{\mathrm{FM}}\right) / \mathrm{Cu}\left(t_{\mathrm{Cu}}\right) /$ $\mathrm{Mn}_{80} \mathrm{Ir}_{20}(10 \mathrm{~nm}) / \mathrm{Ta}(3 \mathrm{~nm})$ were deposited on a Si/ $\mathrm{SiO}_{2}(1000 \mathrm{~nm})$ wafer in a Kurt J. Lesker CMS-18 magnetron sputter system with the easy direction defined along the $x$-direction as described by Østerberg et al. ${ }^{19}$ All combinations of sensor stacks with $t_{\mathrm{FM}}=10,20$, or $30 \mathrm{~nm}$ and $t_{\mathrm{Cu}}=0,0.3$, or $0.6 \mathrm{~nm}$ were fabricated and characterized. Electrical contacts of $\operatorname{Ti}(5 \mathrm{~nm}) / \operatorname{Pt}(100 \mathrm{~nm}) / \mathrm{Au}(100 \mathrm{~nm}) /$ $\mathrm{Ti}(5 \mathrm{~nm})$ were deposited by electron beam evaporation and defined by lift-off. Last, a layer of Ormocomp (micro resist technology GmbH, Berlin, Germany) with a nominal thickness of $1000 \mathrm{~nm}$ was used as a protective layer. Each fabricated chip had both a ring and a diamond sensor to ensure identical stack properties of the two sensors.

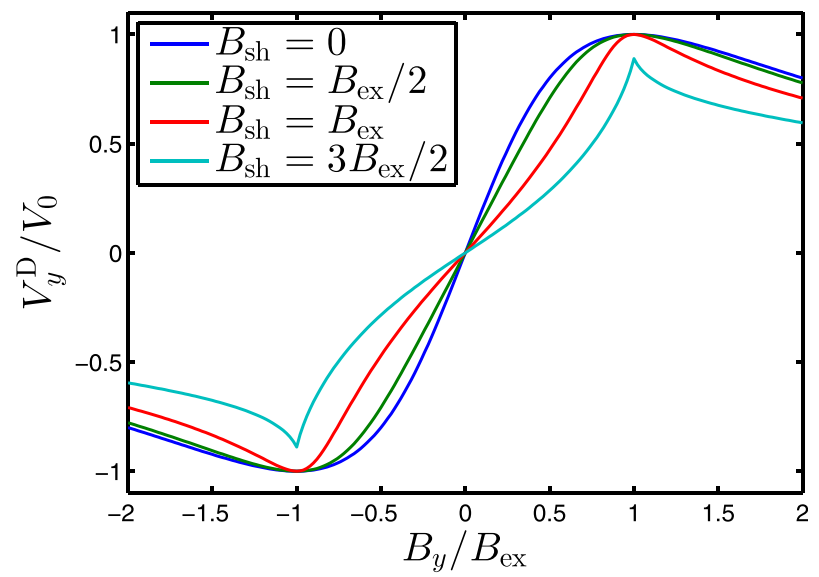

FIG. 2. Response of diamond sensor vs. normalized magnetic field calculated from Eqs. (2) and (3) for increasing shape anisotropy field, $B_{\mathrm{sh}}$. The calculations were performed for $B_{\mathrm{K}}=0$. The signal is normalized to the peak signal obtained for zero shape anisotropy, $V_{0}$.

\section{B. Experimental setup}

A reusable polymethyl methacrylate (PMMA) top provided electrical contact to the chip via spring-loaded pins. ${ }^{21}$ A Peltier element controlled by a LFI-3751 temperature controller (Wavelength Electronics, Inc., MT, USA) kept the sensor at $25.0(1)^{\circ} \mathrm{C}$.

To characterize the sensors, $B_{y}$ was swept in both directions between $\pm 11 \mathrm{mT}$ using a homebuilt Helmholtz coil. The sinusoidal AC sensor bias current $I_{x}$ with a root mean square (RMS) value of $I=1 / \sqrt{2} \mathrm{~mA}$ and a frequency of $f=167 \mathrm{~Hz}$ was provided by a Keithley 6221 current source. The first harmonic in-phase component of the timedependent sensor signal, $V^{\mathrm{R}, \mathrm{D}}$, was recorded using a Stanford Research Systems (SRS) SR830 lock-in amplifier after 100× preamplification by an SRS552 voltage preamplifier. All results below were corrected for the preamplification factor and RMS values are consistently used. The Wheatstone bridge resistance $R_{\mathrm{b}}$ was measured independently with a Keithley 2000 Multimeter.

\section{Fitting of field sweeps}

Both the ring and diamond sensor responses were analyzed using Eqs. (1) and (2) with $\theta_{+}$and $\theta_{-}$determined by numerical minimization of Eq. (3) assuming $\alpha_{ \pm}= \pm 45^{\circ}$. This description was also used for the ring sensors, where $\alpha$ varies along the resistor, to maintain a simple description and to allow for a comparison between the parameters obtained from the fits for the ring and diamond sensors. In the fits, the free parameters were the peak-to-peak signal $V_{\mathrm{pp}}$, a sensor signal offset and the fields $B_{\mathrm{ex}}, B_{\mathrm{K}}$, and $B_{\mathrm{sh}}$. From fits with all parameters free, we found that $B_{\mathrm{K}}$ varied only slightly between the different sensors and stacks and therefore this parameter was fixed to its average value $B_{\mathrm{K}}=0.72 \mathrm{mT}$. Further, the low-field sensitivity $S_{0}$ was extracted as the low-field slope of the fitted curve. Below, parameters are given superscripts $\mathrm{R}$ and $\mathrm{D}$ to indicate the sensor geometry for which they have been obtained.

\section{RESULTS}

\section{A. Field sweeps for different sensors and stacks}

Figure 3 shows the sensor responses measured for diamond and ring sensors that are either bare or surrounded by stack with $t_{\mathrm{FM}}=10,20$, and $30 \mathrm{~nm}$ and $t_{\mathrm{Cu}}=0$ or $0.6 \mathrm{~nm}$. For $t_{\mathrm{FM}}=10 \mathrm{~nm}$, the diamond sensors (red lines) are observed to span a higher signal range, $V_{\mathrm{pp}} / I$, and to have a higher low-field sensitivity, $S_{0}$, than the corresponding ring sensors (blue lines) in accordance with theory. For increasing values of $t_{\mathrm{FM}}$, the low-field sensitivities for the two bare sensor designs become almost identical. For $t_{\mathrm{FM}}=30 \mathrm{~nm}$, the low-field sensitivities for the two sensor shapes are still nearly identical, although the response curves for the bare designs display the $\mathrm{S}$-shape characteristic indicating a significant shape anisotropy (cf. Fig. 2). This effect is less pronounced for the sensors surrounded by stack and hence these are less affected by shape anisotropy.

For increasing $t_{\mathrm{FM}}$ (left to right in Fig. 3) or increasing $t_{\mathrm{Cu}}$ (top to bottom), the magnetic field corresponding to the peak in the response, $B_{\text {peak }}$, decreases (note the different scales 


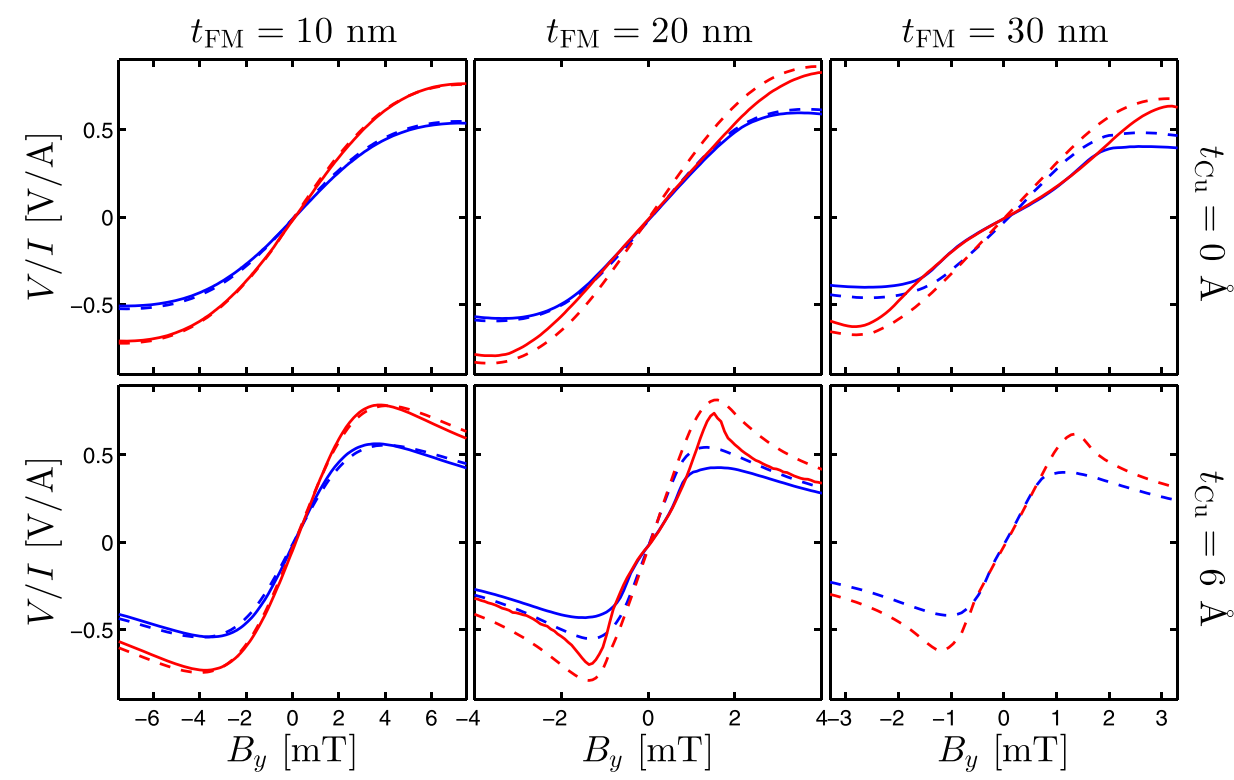

- R, bare ---R, surrounded - D, bare - - - D, surrounded
FIG. 3. Sensor response vs. field for the indicated values of $t_{\mathrm{FM}}$ and $t_{\mathrm{Cu}}$ for ring $(\mathrm{R}$, blue) and diamond $(\mathrm{D}$, red) sensors that are either bare (solid lines) or surrounded by the magnetic stack (dashed lines). Note the different scales on the $B_{y}$ axes. on the field axes). This decrease is caused by the reduction of $B_{\mathrm{ex}}$ with increasing values of $t_{\mathrm{Cu}}$ and $t_{\mathrm{FM}}$ and the resulting weakening of the exchange bias coupling relative to the external magnetic field. For the sensors with low values of $B_{\text {ex }}$, the low-field sensitivity is strongly influenced by the presence of the surrounding stack, and in the extreme case of $t_{\mathrm{FM}}=30$ $\mathrm{nm}$ and $t_{\mathrm{Cu}}=0.6 \mathrm{~nm}$ only the geometries with surrounding stack function as magnetic field sensors. Also, for decreasing $B_{\text {ex }}$, the low-field sensitivity of the diamond sensors decreases towards that of the ring sensors even though the diamond sensors maintain a higher total change in signal, $V_{\mathrm{pp}} / I$. This mismatch between the change in sensitivity and the peak signal is caused by increasing relative importance of shape anisotropy. The results thus indicate that the diamond sensor is affected more by shape anisotropy than the ring sensor.

\section{B. Parameters extracted from fits of field sweeps}

For all fabricated sensors, field sweeps like those in Fig. 3 were measured and fitted according to Sec. III C. Table I lists the values of the peak-to-peak sensor signal $V_{\mathrm{pp}}$, the exchange bias field $B_{\text {ex }}$, and the shape anisotropy field $B_{\text {sh }}$ obtained from the fits as well as the values of the low-field sensitivity $S_{0}$ and the bridge resistance $R_{\mathrm{b}}$.

The values of the bridge resistance, $R_{\mathrm{b}}$, are generally found to be $9(1) \%$ higher for the ring sensors compared to the diamond sensors. The higher ring resistance is independent of the magnetic stack and thus depends only on the geometry in agreement with the theoretical predicted increase of $11 \%$. The slightly lower observed increase may be due to other contributions to the resistance (measured using a 2wire configuration) that reduce the relative difference between the two geometries.

The values of $V_{\mathrm{pp}}$ (given as $V_{\mathrm{pp}} / I$ ) in Table I are independent of whether there is a surrounding stack and are generally found to be about $40 \%$ higher for diamond sensors than for ring sensors. The values of $V_{\mathrm{pp}}$ are found to increase when $t_{\mathrm{FM}}$ is increased from $10 \mathrm{~nm}$ to $20 \mathrm{~nm}$, but they decrease again when $t_{\mathrm{FM}}$ is further increased to $30 \mathrm{~nm}$. For $t_{\mathrm{FM}}=10$ and $20 \mathrm{~nm}, V_{\mathrm{pp}}$ is found to depend only little on

TABLE I. Values of the peak-to-peak sensor signal $V_{\mathrm{pp}}$ (given as $V_{\mathrm{pp}} / I$ ), the exchange bias field $B_{\mathrm{ex}}$, and the shape anisotropy field $B_{\mathrm{sh}}$ obtained from fits of the single domain model to the measured sweeps of the sensor response vs. magnetic field for ring and diamond sensors with the indicated values of $t_{\mathrm{FM}}$ and $t_{\mathrm{Cu}}$. The table also gives the values of the bridge resistance $R_{\mathrm{b}}$ and the low-field sensitivity $S_{0}$. The values to the left and right of the slashes are from bare and surrounded sensors, respectively.

\begin{tabular}{|c|c|c|c|c|c|c|c|c|c|c|c|}
\hline \multirow[b]{2}{*}{$\begin{array}{l}t_{\mathrm{FM}} \\
(\mathrm{nm})\end{array}$} & \multirow[b]{2}{*}{$\begin{array}{c}t_{\mathrm{Cu}} \\
(\mathrm{nm})\end{array}$} & \multicolumn{5}{|c|}{ Ring } & \multicolumn{5}{|c|}{ Diamond } \\
\hline & & $\begin{array}{l}R_{\mathrm{b}}^{\mathrm{R}} \\
(\Omega)\end{array}$ & $\begin{array}{l}V_{\mathrm{pp}}^{\mathrm{R}} / I \\
(\mathrm{~V} / \mathrm{A})\end{array}$ & $\begin{array}{c}B_{\mathrm{ex}}^{\mathrm{R}} \\
(\mathrm{mT})\end{array}$ & $\begin{array}{c}B_{\mathrm{sh}}^{\mathrm{R}} \\
(\mathrm{mT})]\end{array}$ & $\begin{array}{c}S_{0}^{\mathrm{R}} \\
(\mathrm{V} /(\mathrm{A} \mathrm{T}))\end{array}$ & $\begin{array}{l}R_{\mathrm{b}}^{\mathrm{D}} \\
(\Omega)\end{array}$ & $\begin{array}{l}V_{\mathrm{pp}}^{\mathrm{D}} / I \\
(\mathrm{~V} / \mathrm{A})\end{array}$ & $\begin{array}{c}B_{\mathrm{ex}}^{\mathrm{D}} \\
(\mathrm{mT})\end{array}$ & $\begin{array}{c}B_{\mathrm{sh}}^{\mathrm{D}} \\
(\mathrm{mT})\end{array}$ & $\begin{array}{c}S_{0}^{\mathrm{D}} \\
(\mathrm{V} /(\mathrm{A} \mathrm{T}))\end{array}$ \\
\hline 10 & 0.0 & $245 / 254$ & $1.0 / 1.1$ & $6.7 / 6.6$ & $0.6 / 0.0$ & $120 / 130$ & $224 / 230$ & $1.5 / 1.5$ & $6.9 / 6.9$ & $1.2 / 0.6$ & $170 / 170$ \\
\hline 10 & 0.3 & $235 / 241$ & $1.1 / 1.1$ & $3.1 / 3.5$ & $0.5 / 0.4$ & $260 / 230$ & $214 / 220$ & $1.5 / 1.5$ & $3.2 / 3.5$ & $1.1 / 0.9$ & $320 / 310$ \\
\hline 10 & 0.6 & $234 / 230$ & $1.1 / 1.1$ & $1.8 / 1.7$ & $0.2 / 0.0$ & $400 / 460$ & $212 / 209$ & $1.5 / 1.6$ & $1.8 / 1.8$ & $0.8 / 0.3$ & $490 / 570$ \\
\hline 20 & 0.0 & $139 / 141$ & $1.2 / 1.2$ & $3.0 / 3.1$ & $1.1 / 0.6$ & $260 / 280$ & $127 / 130$ & $1.6 / 1.7$ & $3.2 / 3.2$ & $2.5 / 1.5$ & $280 / 350$ \\
\hline 20 & 0.3 & $133 / 133$ & $1.0 / 1.2$ & $1.5 / 1.6$ & $0.7 / 0.3$ & $390 / 470$ & $121 / 121$ & $1.6 / 1.7$ & $1.7 / 1.6$ & $1.8 / 1.1$ & $370 / 540$ \\
\hline 20 & 0.6 & $133 / 131$ & $0.9 / 1.1$ & $1.1 / 0.9$ & $0.4 / 0.3$ & $420 / 660$ & $122 / 120$ & $1.5 / 1.6$ & $1.0 / 1.0$ & $1.4 / 0.8$ & $400 / 720$ \\
\hline 30 & 0.0 & $105 / 108$ & $0.8 / 1.0$ & $2.1 / 2.1$ & $1.7 / 0.5$ & $180 / 290$ & $97 / 100$ & $1.3 / 1.4$ & $2.4 / 2.3$ & $2.8 / 1.7$ & $190 / 320$ \\
\hline 30 & 0.3 & 98/99 & $0.5 / 0.9$ & $1.2 / 0.9$ & $0.8 / 0.4$ & $200 / 480$ & $90 / 92$ & $1.0 / 1.2$ & $0.9 / 1.0$ & $1.8 / 1.0$ & $180 / 490$ \\
\hline 30 & 0.6 & $94 / 96$ & $-/ 0.8$ & $-/ 0.6$ & $-/ 0.3$ & $-/ 580$ & $86 / 88$ & $-/ 1.3$ & $-/ 0.8$ & $-/ 0.9$ & $-/ 5.6$ \\
\hline
\end{tabular}


$t_{\mathrm{Cu}}$. This general observation is attributed to the current flow in the different layers of the magnetic stack when $t_{\mathrm{FM}}$ increases. The resistance decreases with thicker $t_{\mathrm{FM}}$ whereas the effective AMR ratio of the stack decreases when $t_{\mathrm{FM}}$ approaches $10 \mathrm{~nm} .{ }^{13,22}$ For $t_{\mathrm{FM}}=30 \mathrm{~nm}, V_{\mathrm{pp}}$ is reduced when $t_{\mathrm{Cu}}$ increases due to the high relative importance of the shape anisotropy when the exchange bias becomes weak (cf. Fig. 2).

The values obtained for $B_{\text {ex }}$ are essentially unaffected by the sensor geometry and whether the sensor is surrounded by the magnetic stack or not. This shows that the single domain model produces robust results and indicates that it can be used to extract information about the sensor parameters. The values of $B_{\mathrm{ex}}$ are generally found to be inversely proportional to $t_{\mathrm{FM}}$ and to decrease with increasing $t_{\mathrm{Cu}}$ in agreement with the general expected behavior for exchangebiased thin films.

The values obtained for the shape anisotropy field $B_{\text {sh }}$ are generally found to be substantially smaller for sensors surrounded by stack than for the bare sensors. Moreover, the values of $B_{\mathrm{sh}}$ are substantially smaller for ring sensors than for the corresponding diamond sensors. For increasing values of $t_{\mathrm{FM}}$, the value of $B_{\mathrm{sh}}$ is found to increase as expected. ${ }^{23}$ However, for increasing values of $t_{\mathrm{Cu}}$ and a fixed value of $t_{\mathrm{FM}}$, the value of $B_{\mathrm{sh}}$ is found to decrease.

The values of the low-field sensitivity $S_{0}$ are generally found to increase with increasing values of $t_{\mathrm{FM}}$ and $t_{\mathrm{Cu}}$ due to a weakening of the exchange bias coupling. When $B_{\mathrm{sh}}$ is small compared to $B_{\mathrm{ex}}$, primarily for $t_{\mathrm{FM}}=10 \mathrm{~nm}$, the diamond sensors show values of $S_{0}$ that are $20-35 \%$ higher than those obtained for the ring sensors. For $t_{\mathrm{FM}}=20 \mathrm{~nm}$, the values of $S_{0}$ for the diamond sensors are still 10\%-25\% higher than those for the corresponding ring sensors when the sensor is surrounded by magnetic stack, but when the sensors are bare, $S_{0}$ for the two sensor types are nearly identical. For $t_{\mathrm{FM}}=30 \mathrm{~nm}, S_{0}$ for the two sensor types are approximately the same irrespective of whether the sensor is surrounded by stack or not. The highest low-field sensitivities are obtained for sensors surrounded by stack with $t_{\mathrm{FM}}=20 \mathrm{~nm}$ and $t_{\mathrm{Cu}}=0.6 \mathrm{~nm}$ for both sensor geometries.

\section{Comparison of ring and diamond sensors}

Comparing diamond to ring geometries in Table I, the expected increase in $V_{\mathrm{pp}}$ occurs for all sensors, but the increase in $S_{0}$ is only observed for sensors with comparatively small shape anisotropy. To further investigate the effect of shape anisotropy, Fig. 4 shows $V_{\mathrm{pp}}^{\mathrm{D}} / V_{\mathrm{pp}}^{\mathrm{R}}$ and $S_{0}^{\mathrm{D}} / S_{0}^{\mathrm{R}}$ as function of the relative importance of the shape anisotropy for the diamond sensors, $B_{\mathrm{sh}}^{\mathrm{D}} /\left(B_{\mathrm{ex}}^{\mathrm{D}}+B_{\mathrm{K}}^{\mathrm{D}}\right)$. The figure shows all data from Table I, i.e., values obtained for $t_{\mathrm{FM}}=10,20$, and $30 \mathrm{~nm}$ and $t_{\mathrm{Cu}}=0,0.3$, and $0.6 \mathrm{~nm}$ for both bare sensors and sensors surrounded by stack. The consistency of the points in Fig. 4 show that the behavior is determined by $B_{\mathrm{sh}}^{\mathrm{D}} /\left(B_{\mathrm{ex}}^{\mathrm{D}}+B_{\mathrm{K}}^{\mathrm{D}}\right)$ irrespective of the stack composition and of whether there is a surrounding stack or not. Hence, the results are of general character for the two sensor geometries. The dashed lines in the figure indicate the theoretical prediction of a $\sqrt{2}$ ratio obtained for negligible shape

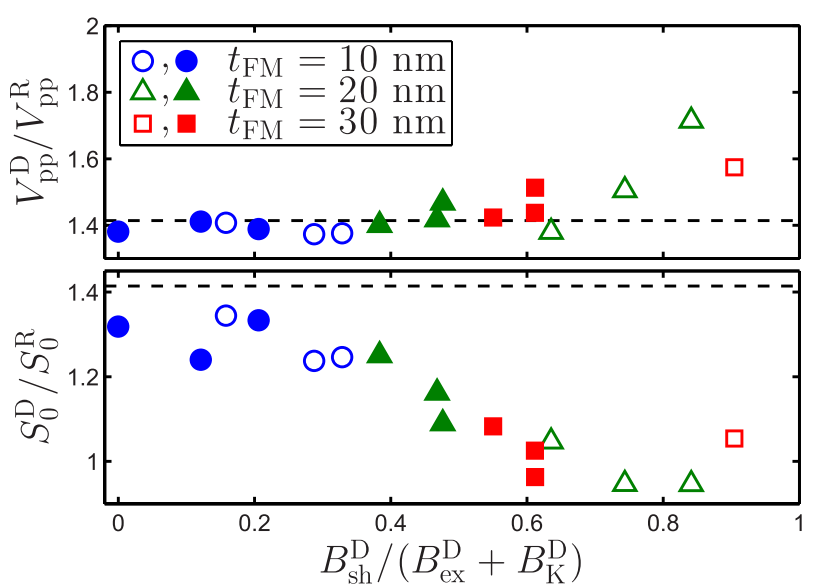

FIG. 4. Ratio of peak-to-peak sensor responses (top) and low-field sensitivities (bottom) for the diamond and ring geometries vs. the relative shape anisotropy obtained for diamond sensors that are bare (open symbols) or surrounded by stack (filled symbols) for the indicated values of $t_{\mathrm{FM}}$. Results for $t_{\mathrm{Cu}}=0,0.3$, and $0.6 \mathrm{~nm}$ are included but not differentiated in the figure. The dashed lines indicate the ratios of $\sqrt{2}$ predicted for negligible shape anisotropy.

anisotropy. Generally, the diamond sensors show the predicted improvement of $V_{\mathrm{pp}}$ when $B_{\mathrm{sh}}^{\mathrm{D}} /\left(B_{\mathrm{ex}}^{\mathrm{D}}+B_{\mathrm{K}}^{\mathrm{D}}\right)<0.6$ and the value of this ratio even increases beyond this value for higher values of $B_{\mathrm{sh}}^{\mathrm{D}} /\left(B_{\mathrm{ex}}^{\mathrm{D}}+B_{\mathrm{K}}^{\mathrm{D}}\right)$. Likewise, the diamond sensors generally have a higher sensitivity than the ring sensors with a sensitivity ratio starting at around 1.3 that decreases monotonically towards 1 when $B_{\mathrm{sh}}^{\mathrm{D}} /\left(B_{\mathrm{ex}}^{\mathrm{D}}+B_{\mathrm{K}}^{\mathrm{D}}\right)$ increases towards 0.6 .

\section{DISCUSSION}

The theory for negligible shape anisotropy predicts the diamond sensors to be $41 \%$ more sensitive than ring sensors for the same bias current. While $41 \%$ improvement was not observed in the results, diamond sensors with low shape anisotropy were about $30 \%$ more sensitive than corresponding ring sensors. However, the diamond sensors were more affected by demagnetization effects than ring sensors such that diamond and ring sensors had similar low-field sensitivities for high relative shape anisotropy.

To further investigate why ring sensors are less affected by shape anisotropy, we calculate the demagnetization field for ring and diamond geometries under the simplifying assumption that the stack is homogeneously magnetized along the $x$-direction. The calculations were carried out using the magnetostatic fields module in the COMSOL Multiphysics software. Figure 5 shows this demagnetization field along with a plot of the absolute sensitivity of the sensor resistance to a small change in magnetization angle $\theta$, which is proportional to $|\sin (2 \alpha)|{ }^{14}$

The top and bottom parts of Fig. 5 show the sensitivity and demagnetization field maps for the two sensor shapes, where black and white indicate maximum and minimum values, respectively. For the diamond sensor, the sensitivity to a change in $\theta$ is maximal and the demagnetization field is of medium magnitude in the entire sensor area. For the ring sensor, the sensitivity to a change in $\theta$ is maximal in the middle part of the ring section, while the outermost parts of the 

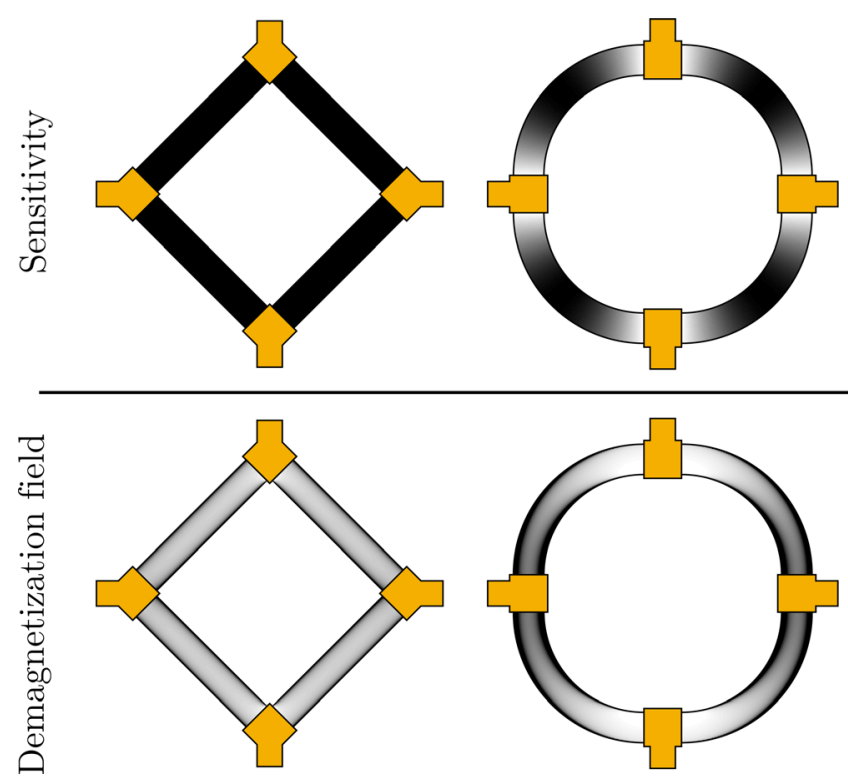

FIG. 5. Spatial distributions of (top) the sensitivity of the bridge output to a small change in the magnetization orientation, and (bottom) the magnitude of the demagnetizing field when the sensor is uniformly magnetized along the $x$-direction. Black indicates high sensitivity and high demagnetization field, whereas white indicates zero values. The same scales are used for the diamond and ring sensors.

ring close to the contacts are essentially insensitive to a change in $\theta$. The latter parts essentially add to the overall resistance without contributing to the signal. Correspondingly, the demagnetization field is maximal near the vertical sections of the ring and zero near the horizontal sections of the ring.

For the diamond geometry, the demagnetization field (Fig. 5, bottom) is strongest at the edge of the resistors, while for the ring geometry the demagnetization field is strongest near the current contacts. However, the ring sensor is most sensitive in the middle of the resistors, away from the area of highest demagnetization (Fig. 5, top).

In Table I, the values of $B_{\text {sh }}$ resulting from fits to a single domain description of the magnetic energy are about a factor of two smaller for the ring sensors than for the diamond sensors and concurrently Fig. 4 shows that the ring sensors are significantly less affected by shape anisotropy. This difference must be caused by the interplay between the locations of the most sensitive sensor area and the varying demagnetization field along the ring sensor. In Table I, for fixed $t_{\mathrm{FM}}$, we also observed that $B_{\mathrm{sh}}$ decreased for increasing $t_{\mathrm{Cu}}$. This observation cannot be understood in terms of a homogeneously magnetized single magnetic domain as the nominal shape anisotropy in this case would be independent on $t_{\mathrm{Cu}}$.

A more realistic magnetization configuration would have to allow for a relaxation of the magnetization state away from the nominal single domain orientation to favor a local alignment along the sensor edge to reduce the overall magnetostatic energy. ${ }^{24}$ To further investigate this, we have performed micromagnetic simulations using the OOMMF software $^{25}$ to obtain the magnetization configuration of the diamond sensor in zero external magnetic field for different values of the exchange bias field $B_{\text {ex }}$. The calculations were carried out with periodic boundary conditions ${ }^{26}$ on a domain consisting of a single row of cubic micromagnetic cells with a dimension of $10 \mathrm{~nm}$ corresponding to the film thickness in the simulation. We further used a saturation flux density of $\mu_{0} M_{\mathrm{s}}=1.05 \mathrm{~T}$, an exchange stiffness of $A=13 \times 10^{-12} \mathrm{~J} / \mathrm{m}$, and a uniaxial anisotropy corresponding to $B_{\mathrm{K}}=0.72 \mathrm{mT}$. Figure 6 shows the magnetization orientation $\theta$ in zero external magnetic field as function of the distance $\delta$ from the sensor edge for a sensor element oriented at an angle of $45^{\circ}$ to the $x$-direction, which also defines the exchange bias direction. The nominal magnetization orientation in zero magnetic field is $\theta=0^{\circ}$. Figure 6 clearly shows that the magnetization orientation relaxes in a narrow region near the sensor edge and approaches an orientation along the sensor edge when $\delta$ approaches zero. The distance over which this relaxation takes place is clearly observed to decrease with increasing $B_{\mathrm{ex}}$. The corresponding average magnetostatic energy density $u_{\mathrm{ms}}=-\mu_{0}\left\langle\boldsymbol{M} \cdot \boldsymbol{H}_{\mathrm{d}}\right\rangle$, where the local values of the magnetization and demagnetization field are averaged over the volume of the structure, shown in the inset of Fig. 6, is observed to be reduced when $B_{\mathrm{ex}}$ is lowered. This shows that when the exchange bias field is low, it is easier for the local relaxation of the magnetization to take place and thus the equivalent single domain shape anisotropy is reduced.

This mechanism at least qualitatively explains the experimental observation of a reduction of the equivalent single domain shape anisotropy $B_{\mathrm{sh}}$ for increasing $t_{\mathrm{Cu}}$ and a fixed value of $t_{\mathrm{FM}}$ for both sensor geometries. The failure of both bare sensor geometries for $t_{\mathrm{FM}}=30 \mathrm{~nm}$ and $t_{\mathrm{Cu}}=0.6 \mathrm{~nm}$ shows that a too weak exchange bias compared to the shape anisotropy will render the sensors unusable.

The relaxation of the edge magnetic configuration for increasing shape anisotropy also reduces the observed value of $V_{\mathrm{pp}}$ as a progressively increasing fraction of the crosssection of a sensor branch will have a magnetization orientation pinned parallel to the sensor edge. The results in Table I

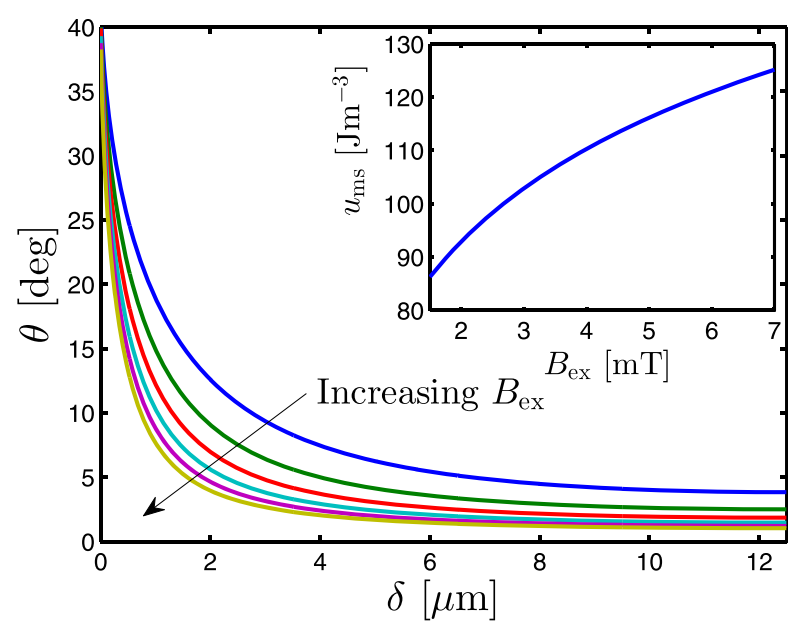

FIG. 6. (a) Result of micromagnetic calculation of the zero-field magnetization orientation $\theta$ vs. separation $\delta$ from the edge of the $25 \mu \mathrm{m}$ wide sensor element with $t_{\mathrm{FM}}=10 \mathrm{~nm}$. The sensor element is oriented at an angle of $45^{\circ}$ $\left(=\alpha_{+}\right)$to the $x$-direction and is exchange-biased along the $x$-direction with exchange bias fields of $B_{\mathrm{ex}}=1.5,2.6,3.7,4.8,5.9$, and $7 \mathrm{mT}$, respectively. The inset shows the corresponding average magnetostatic energy density $u_{\mathrm{ms}}$ as a function of $B_{\mathrm{ex}}$. 
show that the reduction of $V_{\mathrm{pp}}$ for increasing shape anisotropy is higher for the ring sensor than for the diamond sensor. Thus, the two sensor designs are again affected quite differently by the shape anisotropy.

Further work to shed light on the detailed mechanism and the difference between the two sensor geometries should involve micromagnetic simulations of the magnetization state of the sensor structures combined with a calculation of the electrical transport properties. As the entire geometry of a branch of the ring sensor would have to be considered, such calculations would be highly computer demanding and clearly beyond the scope of the present work.

\section{CONCLUSION}

We have performed a systematic experimental comparison of ring and diamond shaped geometries of planar Hall effect sensor bridges with the stack structure $\mathrm{Ni}_{80} \mathrm{Fe}_{20}\left(t_{\mathrm{FM}}\right) /$ $\mathrm{Cu}\left(t_{\mathrm{Cu}}\right) / \mathrm{Mn}_{80} \mathrm{Ir}_{20}(10 \mathrm{~nm})$ with $t_{\mathrm{FM}}=10,20$, and $30 \mathrm{~nm}$ and $t_{\mathrm{Cu}}=0,0.3$, and $0.6 \mathrm{~nm}$. Both bare sensors and sensors surrounded by the magnetic stack with a gap of $3 \mu \mathrm{m}$ were investigated. We investigated whether the theoretical prediction, for negligible shape anisotropy, that the diamond sensors produce a $41 \%$ higher signal than the corresponding ring sensors could be observed experimentally.

For this comparison, we measured and analyzed field sweeps of the sensor response using a single domain model. Our investigation showed that diamond sensors exhibited about $40 \%$ higher change in peak-to-peak signal and about $30 \%$ higher low-field sensitivity compared to ring sensors when the equivalent single domain shape anisotropy field fulfilled $B_{\mathrm{sh}}^{\mathrm{D}}<0.4\left(B_{\mathrm{ex}}^{\mathrm{D}}+B_{\mathrm{K}}^{\mathrm{D}}\right)$. For larger values of $B_{\mathrm{sh}}^{\mathrm{D}}$, the two sensor designs had similar low-field sensitivities and for increasing $B_{\mathrm{sh}}^{\mathrm{D}}$ the peak-to-peak signal change decreased more for the ring sensor than for the diamond sensor. The highest low-field sensitivities were found for $t_{\mathrm{FM}}=20 \mathrm{~nm}$ and $t_{\mathrm{Cu}}=0.6 \mathrm{~nm}$ for sensors surrounded by magnetic stack to be $S_{0}^{\mathrm{D}}=0.72 \Omega / \mathrm{mT}$ for the diamond sensor and $S_{0}^{\mathrm{R}}=0.66 \Omega / \mathrm{mT}$ for the ring sensor. Moreover, due to their shorter length, the diamond sensors have a bridge resistance, which is about $10 \%$ lower than that of the ring sensors. Finally, the diamond sensors have a constant field sensitivity along the length of a branch, whereas the field sensitivity varies along the branch for the ring sensors. This may be disadvantageous for the use of the sensors for biosensing applications.

Our results indicate that the low-field sensitivity of the ring sensors is less affected by shape anisotropy and a relaxation away from the nominal single domain configuration than that of the diamond sensors. The behavior of the ring sensor is the result of a surprisingly rich and complex interplay between demagnetization effects/magnetization relaxation and the electrical transport properties. Our results indicate that the effect of shape anisotropy can be significantly reduced by letting the magnetic stack surround the sensor geometry with a small gap (in our case $3 \mu \mathrm{m}$ ). Suggestions for further work on micromagnetic calculations combined with electrical transport calculations to shed light on the shape anisotropy effects were proposed.

\section{ACKNOWLEDGMENTS}

This work was supported by the Danish Council for Independent Research (Postdoc Project No. DFF-400500116).

${ }^{1}$ S. X. Wang and G. Li, "Advances in giant magnetoresistance biosensors with magnetic nanoparticle tags: Review and outlook," IEEE Trans. Magn. 44, 1687-1702 (2008).

${ }^{2}$ P. P. Freitas, R. Ferreira, S. Cardoso, and F. Cardoso, "Magnetic sensors and their applications," J. Phys.: Condens. Matter 19, 165221 (2007).

${ }^{3}$ J. S. Moodera, L. R. Kinder, T. M. Wong, and R. Meservey, "Large magnetoresistance at room temperature in ferromagnetic thin film tunnel junctions," Phys. Rev. Lett. 74(16), 3273 (1995).

${ }^{4}$ S. G. Grancharov, H. Zeng, S. Sun, S. X. Wang, S. O’Brien, C. B. Murray, J. R. Kirtley, and G. A. Held, "Bio-functionalization of monodisperse magnetic nanoparticles and their use as biomolecular labels in a magnetic tunnel junction based sensor," J. Phys. Chem. B 109(26), 13030-13035 (2005).

${ }^{5}$ L. Ejsing, M. F. Hansen, A. K. Menon, H. A. Ferreira, D. L. Graham, and P. P. Freitas, "Planar Hall effect sensor for magnetic micro- and nanobead detection," Appl. Phys. Lett. 84(23), 4729 (2004).

${ }^{6}$ S. Oh, P. B. Patil, T. Q. Hung, B. Lim, M. Takahashi, D. Y. Kim, and C. G. Kim, "Hybrid AMR/PHR ring sensor," Solid State Commun. 151(18), 1248-1251 (2011).

${ }^{7}$ B. Sinha, T. Q. Hung, T. S. Ramulu, S. Oh, K. Kim, D. Y. Kim, F. Terki, and C. G. Kim, "Planar Hall resistance ring sensor based on NiFe/Cu/IrMn trilayer structure,” J. Appl. Phys. 113(6), 063903 (2013).

${ }^{8}$ N. T. Thanh, B. Parvatheeswara Rao, N. H. Duc, and C. G. Kim, "Planar Hall resistance sensor for biochip application," Phys. Status Solidi A 204(12), 4053-4057 (2007).

${ }^{9}$ D. Issadore, Y. I. Park, H. Shao, C. Min, K. Lee, M. Liong, R. Weissleder, and H. Lee, "Magnetic sensing technology for molecular analyses," Lab Chip 14(14), 2385-2397 (2014).

${ }^{10}$ J. Lenz and A. S. Edelstein, "Magnetic sensors and their applications," IEEE Sens. J. 6(3), 631 (2006).

${ }^{11}$ C. D. Damsgaard, S. C. Freitas, P. P. Freitas, and M. F. Hansen, "Exchange-biased planar Hall effect sensor optimized for biosensor applications," J. Appl. Phys. 103(7), 07 A302 (2008).

${ }^{12}$ T. Q. Hung, S. Oh, J. R. Jeong, and C. G. Kim, "Spin-valve planar Hall sensor for single bead detection," Sens. Actuators, A 157(1), 42-46 (2010).

${ }^{13}$ T. Q. Hung, S. Oh, S. Anandakumar, J. R. Jeong, D. Y. Kim, and C. Kim, "Optimization of the multilayer structures for a high field-sensitivity biochip sensor based on the planar Hall effect," IEEE Trans. Magn. 45(10), 4518-4521 (2009).

${ }^{14}$ A. D. Henriksen, B. T. Dalslet, D. H. Skieller, K. H. Lee, F. Okkels, and M. F. Hansen, "Planar Hall effect bridge magnetic field sensors," Appl. Phys. Lett. 97(1), 013507 (2010).

${ }^{15}$ B. Sinha, S. Oh, T. Sri Ramulu, J. Lim, D. Y. Kim, and C. G. Kim, "Planar Hall effect ring sensors for high field-sensitivity," Adv. Mater. Res. 317, 1136-1140 (2011).

${ }^{16}$ F. W. Østerberg, G. Rizzi, A. D. Henriksen, and M. F. Hansen, "Planar Hall effect bridge geometries optimized for magnetic bead detection," J. Appl. Phys. 115(18), 184505 (2014).

${ }^{17}$ G. Rizzi, F. W. Østerberg, M. Dufva, and M. F. Hansen, "Magnetoresistive sensor for real-time single nucleotide polymorphism genotyping," Biosens. Bioelectron. 52, 445-451 (2014).

${ }^{18}$ F. W. Østerberg, G. Rizzi, M. Donolato, R. S. Bejhed, A. Mezger, M. Strömberg, M. Nilsson, M. Strømme, P. Svedlindh, and M. F. Hansen, "Onchip detection of rolling circle amplified DNA molecules from Bacillus Globigii spores and Vibrio Cholerae," Small 10(14), 2877-2882 (2014).

${ }^{19}$ F. W. Østerberg, G. Rizzi, T. Zardán Gómez de la Torre, M. Strömberg, M. Strømme, P. Svedlindh, and M. F. Hansen, "Measurements of Brownian relaxation of magnetic nanobeads using planar Hall effect bridge sensors," Biosens. Bioelectron. 40(1), 147-152 (2013).

${ }^{20}$ F. W. Østerberg, A. D. Henriksen, G. Rizzi, and M. F. Hansen, "Comment on "Planar Hall resistance ring sensor based on $\mathrm{NiFe} / \mathrm{Cu} / \mathrm{IrMn}$ trilayer structure [J. Appl. Phys. 113, 063903 (2013)],"’’ J. Appl. Phys. 114(10), 106101 (2013).

${ }^{21}$ A. D. Henriksen, G. Rizzi, F. W. Østerberg, and M. F. Hansen, "Optimization of magnetoresistive sensor current for on-chip magnetic bead detection using the sensor self-field," J. Magn. Magn. Mater. 380, 209-214 (2014). 
${ }^{22}$ V. Gehanno, P. P. Freitas, A. Veloso, J. Ferreira, B. Almeida, J. B. Sousa, A. Kling, J. C. Soares, and M. F. da Silva, "Ion beam deposition of Mn-Ir spin valves," IEEE Trans. Magn. 35, 4361-4367 (1999).

${ }^{23}$ M. Beleggia, M. De Graef, and Y. T. Millev, "The equivalent ellipsoid of a magnetized body," J. Phys. D: Appl. Phys. 39(5), 891 (2006).

${ }^{24}$ M. Donolato, B. T. Dalslet, C. D. Damsgaard, K. Gunnarsson, C. S. Jacobsen, P. Svedlindh, and M. F. Hansen, "Size-dependent effects in exchange-biased planar Hall effect sensor crosses," J. Appl. Phys. 109, 064511 (2011).

${ }^{25}$ See http://math.nist.gov/oommf/ for object oriented micromagnetic framework (oommf), version 1.2.

${ }^{26}$ K. M. Lebecki, M. J. Donahue, and M. W. Gutowski, "Periodic boundary conditions for demagnetization interactions in micromagnetic simulations,” J. Phys. D: Appl. Phys. 41, 175005 (2008). 